Research Article

\title{
Properties of spectrally bounded compact elementary operators
}

\author{
Willy Kangogo*, Nyaare Benard Okelo, Omolo Ongati \\ Department of Pure and Applied Mathematics, Jaramogi Oginga Odinga University of Science and Technology, Bondo, Kenya
}

(Received: 15 February 2021. Received in revised form: 29 March 2021. Accepted: 30 March 2021. Published online: 31 March 2021.)

(c) 2021 the authors. This is an open access article under the CC BY (International 4.0) license (www.creativecommons.org/licenses/by/4.0/).

\begin{abstract}
Spectrally bounded compact elementary operators on dense irreducible subalgebras of $C^{*}$-algebras are characterized. Also, it is shown that left multiplications, right multiplications, generalized derivations and basic elementary operators are spectrally bounded compact elementary operators. Furthermore, several properties of spectrally bounded compact elementary operators such as completeness, convergence, continuity and total boundedness in a general Banach setting are outlined.
\end{abstract}

Keywords: elementary operator; spectral boundedness; irreducible; spectral operator norm.

2020 Mathematics Subject Classification: 47B47, 47A30.

\section{Introduction}

The study of elementary operators has become an active area of research in operator theory [2]. The elementary operator, being an operator on the Banach space $B(H)$, has attracted much attention of many mathematicians. Some results about the spectra, the numerical ranges and norms of elementary operators were studied in [2,6]. Actually, the norm of an elementary operator is traced back to the works of Stampli [30] who worked on the norms of derivations. In [8], the norm of an elementary operator was determined to be

$$
\sup \left\{\left\|\sum_{i=1}^{n} A_{i} X B_{i}\right\| ; X \in B(H) ;\|X\| \leq 1\right\}=\sup \left\{\left\|\sum_{i=1}^{n} A_{i} U B_{i}\right\|: U U^{*}=U^{*} U=I ; U \in B(H)\right\} .
$$

In addition, Okelo [22] determined the norm a two-sided multiplication operator on $C^{*}$-algebra. He showed that for any complex Hilbert space $H$, if $T_{A, B}: B(H) \rightarrow B(H)$ such that $T_{A, B}(X)=A X B \quad \forall X \in B(H)$ then $\left\|T_{A, B}(X)\right\|=\|A\|\|B\|$. Furthermore, Okelo [23] investigated the orthogonality of elementary operators and gave the necessary and sufficient conditions for their normality. Also, in [24] the author considered the orthogonality of elementary operators when they are implemented by norm-attainable operators on a Hilbert space. In particular, the study of elementary operators has been developed in two branches; namely, spectral properties and structural properties. Therefore, a systematic study of elementary operators was started by Lumer and Rosenblum [14] who studied the spectral properties of elementary operators. Later, Vala [32] pioneered the study of compactness of elementary operators and proved that a linear mapping $T \mapsto A T B$ is compact if and only if $A$ and $B$ are compact operators on a Banach space. In connection to compactness of operators, Akemann and Wright [1] gave the necessary and sufficient conditions for a given $C^{*}$-algebra to admit a nonzero compact or weakly compact derivations; that is, a $C^{*}$-algebra admits a non-zero compact derivations if and only if it contains nonzero finite dimensional central projections. Fong and Sourour [10] characterized compactness of elementary operators on $B(H)$, where $B(H)$ is set of all bounded linear operators on separable Hilbert Space $H$, and they showed that an elementary operator on $B(H)$ is compact if and only if it has representation $T_{A, B}(X)=\sum_{i=1}^{n} A_{i} X B_{i}$ for $A_{i}$ and $B_{i}$ being compact. Saksman and Tylli [27] studied a number of structural properties of elementary operators on Banach space setting such as basic sequence techniques applied in Banach spaces and weak compactness or strict singularity of a basic two sided multiplication operator. These properties include the norms and spectra of elementary operators in various settings. A detailed study of spectral boundedness of elementary operators was introduced by Mathieu [15] in which the results were motivated by their connections to the non-commutative Singer-Wermer conjuncture and Kaplansky's problem of invertibility-preserving operators. Bresar and Turovskii [3] studied elementary operators on Banach algebras that are simultaneously compact and they considered an algebra $\mathcal{A}$ over a field $\mathbb{K}$, where $A \in \mathcal{A}$ is of completely finite rank if both $L_{A}$ and $R_{A}$ are finite rank. Denote by $A_{c f}$ the set of all completely finite rank elements of $\mathcal{A}$. In [3], it was shown that if 
$A_{c f}$ is the sum of all finite dimensional ideals of $\mathcal{A}$, then every element in $\mathcal{A}_{c f}$ generates a finite dimensional ideal in $\mathcal{A}$. Fialkow [9] presented a number of results on spectral properties of elementary operators where a new expression for the essential spectra of an elementary operator was derived, that is, $\sigma_{e}\left(R_{A B}\right)=\sigma_{H}(A) \circ \sigma_{H_{e}}(B) \cup \sigma_{H_{e}}(A) \circ \sigma_{H}(B)$. Shulmann and Turowska [29] discussed properties of operators on Hilbert spaces which share similar properties with elementary operators on $B(H)$. This is because $T_{A, B}(X)=\sum_{k=1}^{n} L_{A_{k}} R_{B_{k}}$ has a formal adjoint $T_{A^{*}, B^{*}}^{*}(X)=\sum_{k=1}^{n} L_{A_{k}^{*}} R_{B_{k}^{*}}$ which turns into a proper adjoint if restricted to the ideal $S_{2}$ of Hilbert Schmidt operators. These properties include approximate intertwinings, the intersection of the kernel of adjoint, ranges of derivations and commutators, spectral subspaces and Fuglede type theorems among others.

The study of positive maps in operator theory has also attracted considerable attention from many researchers. Huruya and Tomiyama [20] studied completely bounded maps of $C^{*}$-algebras and proved that a completely positive map is completely bounded. For example, a derivation is completely bounded but not completely positive. Also, every bounded linear map of $C^{*}$-algebra into a commutative $C^{*}$-algebra is completely bounded. Let $\mathcal{A}$ and $\mathcal{B}$ be a $C^{*}$-algebras, it is shown [20] that for a fixed $C^{*}$-algebra $\mathcal{A}$, every bounded linear map of $\mathcal{A}$ into an arbitrary $C^{*}$-algebra $\mathcal{B}$ is completely bounded if and only if $\mathcal{A}$ finite dimensional. Furthermore, Huruya and Tomiyama [20] proved that for a fixed $C^{*}$-algebra $\mathcal{B}$ every bounded linear map of an arbitrary $C^{*}$-algebra $\mathcal{A}$ into $\mathcal{B}$ is completely bounded if and only if every irreducible representation of $\mathcal{B}$ is finite dimensional with bounded degree. Curto and Mathieu [5] gave a characterization of spectral boundedness of generalized inner derivation on unital Banach algebra to be spectrally bounded. Furthermore, Mathieu [17] showed that a derivation on a Banach algebra $\mathcal{A}$ is spectrally bounded if and only if it maps it into $\operatorname{Rad} \mathcal{A}$. Mathieu [19] gave a review on the recent advances in the study of elementary operators that have not been explored before. The main question being when an elementary operator is spectrally bounded or spectrally isometric. Therefore, the study of spectral characterizations of operators has attracted a lot of research in past years. In [16], it was shown that every spectrally bounded operators need not to be continuous in general hence it is important to study spectral boundedness of compact elementary operators on dense irreducible subalgebras. Semrl [28] studied spectrally bounded linear maps on $B(H)$ and showed that if $H$ is a separable infinite dimensional Hilbert space, then $T: B(H) \rightarrow B(H)$ is a unital surjective linear spectrally bounded map which is either an automorphism or anti-automorphism. Furthermore, Lin and Mathieu [13] proved that every unital bounded linear mapping from a purely infinite $C^{*}$-algebra of real rank zero into a unital Banach algebra which preserves elements of square zero is a Jordan homomorphism. In the results, they used unital surjective spectral isometries as Jordan homomorphisms.

As we have seen that a lot of research has been done on the structural properties of elementary operators but there are still open problems on spectral boundedness of compact elementary operators on dense irreducible subalgebras. In this paper, we characterize spectral boundedness of compact elementary operators on dense irreducible subalgebras.

\section{Preliminaries}

In this section, we outline preliminary concepts which are useful in the sequel. Let $\mathcal{A}$ be a Banach algebra. We denote a

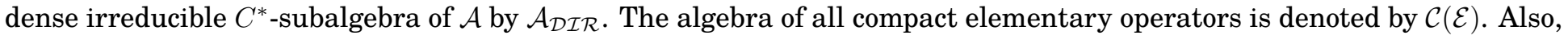
we denote the algebra of all spectrally bounded compact elementary operators by $\mathcal{C}_{\mathcal{S B D}}(\mathcal{E})$.

Definition 2.1. ([21], Section 2) Consider a $C^{*}$-algebra $\mathcal{A}$ and let $T: \mathcal{A} \rightarrow \mathcal{A}, T$ is called an elementary operator if it has the following representation: $T_{A, B}(X)=\sum_{i=1}^{n} A_{i} X B_{i} \forall A_{i}, B_{i}$ are fixed in $\mathcal{A}$ or $\mathcal{M}(\mathcal{A})$ is multiplier algebra of $\mathcal{A}$. For $A, B \in B(H)$, we define particular elementary operators as follows:

(i). The left multiplication operator $L_{A}: B(H) \rightarrow B(H)$ by $L_{A}(X)=A X, \forall X \in B(H)$.

(ii). The right multiplication operator $R_{B}: B(H) \rightarrow B(H)$ by $R_{B}(X)=X B, \forall X \in B(H)$.

(iii). The generalized derivation (implemented by $A, B$ ) by $\delta_{A, B}(X)=L_{A}-R_{B}, \forall X \in B(H)$.

(iv). The basic elementary operator (implemented by $A, B$ ) by $M_{A, B}(X)=A X B, \forall X \in B(H)$.

(v). The Jordan elementary operator (implemented by $A, B)$ by $\mathcal{U}_{A, B}(X)=A X B+B X A, \forall X \in B(H)$.

Definition 2.2. [12] Let $V$ be a linear vector space. A non-negative real valued function $\|\|:. V \rightarrow \mathbb{R}$ is called a norm on $V$ if it satisfies the following conditions:

(i). $\|a\| \geq 0$ and $\|a\|=0$ if and only if $a=0$ for all $a \in V$.

(ii). $\|\alpha a\|=|\alpha|\|a\|$ for all $a \in V$ and $\alpha \in \mathbb{K}$. 
(iii). $\|a+b\| \leq\|a\|+\|b\|$ for all $a, b \in V$.

The ordered pair $(V,\|\|$.$) is called a normed space.$

Definition 2.3. ([4], Definition 4.1) If $\mathcal{A}$ is any $C^{*}$-algebra and $\Phi: \mathcal{A} \rightarrow B(H)$ is a representation then $\Phi$ is irreducible if $\Phi(\mathcal{A})$ is an irreducible subalgebra of $\mathcal{B}(\mathcal{H})$.

Definition 2.4. ([11], Definition 2.2) Let $\mathcal{A}$ and $\mathcal{B}$ be complex Banach algebras. A linear mapping $T: \mathcal{A} \rightarrow \mathcal{B}$ is called spectrally bounded if there exists a constant $M \geq 0$ such that $r(T x) \leq \operatorname{Mr}(x)$ and spectrally infinitesimal if $r(T(x))=0$, for all $x \in \mathcal{A}$. If $r(T(x))=r(x)$, for all $x \in \mathcal{A}$ we say that $T$ is a spectral isometry. If $r(x)=0$, then $x$ is called quasi-nilpotent.

Definition 2.5. ([31], Definition 1.3) A Banach space $\Omega$ is said to have the approximation property for every compact subset $C$ of $\Omega$ and for every $\varepsilon>0$ there exists a finite rank operator $T \in B(\Omega)$ such that $\|T x-x\|<\varepsilon$ for each $x \in C$.

Definition 2.6. ([7], Proposition 2.2.1) The radical of an algebra $\mathcal{A}$ is defined to be intersection of the maximal left ideals of $\mathcal{A}$ denoted by rad $\mathcal{A}$. An algebra (non-unital) is radical if rad $\mathcal{A}=0$ then $\operatorname{rad} \mathcal{A}$ is an ideal in $\mathcal{A}$ and $\mathcal{A} / \mathrm{rad}(\mathcal{A})$ is a semi-simple algebra.

Remark 2.1. An elementary operator leaves each ideal of $\mathcal{A}$ invariant hence it is close at hand to use primitive ideals and dense algebras of operators to study properties of it [6].

Definition 2.7. ([25], Definition 4.2.12) A representation $T$ of an algebra $\mathcal{A}$ on a linear space $X$ is called strictly dense if whenever $x_{1}, x_{2}, \ldots, x_{n}$ is a finite list of linearly independent vectors in $X$ and $y_{1}, y_{2}, \ldots, y_{n}$ is a list of vectors in $X$ then there is an element $a \in A$ with $T_{a} x_{j}=y_{j}$ for $j=1,2, \ldots, n$.

Definition 2.8. ([25], Definition 4.1.17) Let $\mathcal{A}$ be an algebra and let $X$ be a linear space. Then $X$ is called an $\mathcal{A}$-module if there is a fixed representation $T: \mathcal{A} \rightarrow B(X)$ and $T_{a} x$ denoted by ax, $a \in \mathcal{A}$ and $x \in X$. If $\mathcal{A}$ and $X$ are both normed and the representation $T: \mathcal{A} \rightarrow B(X)$ is continuous then the corresponding module is called a left $\mathcal{A}$-module.

Remark 2.2. If $X$ is a both a left and right $\mathcal{A}$-module and if the two module actions satisfy $a(x b)=(a x) b \forall \mathcal{A} x \in X$ then is called $\mathcal{A}$-bimodule [25].

Definition 2.9. Let $T_{A_{i}, B_{i}} \in \mathcal{C}(\mathcal{E})$ for the set of all spectrally bounded compact operators. We define

$$
\left\|T_{A_{i}, B_{i}}\right\|_{\sigma}=\inf \left\{M \geq 0\left|r\left(T_{A_{i}, B_{i}}(X)\right)\right| \leq M r(X)\right\},
$$

for all $X \in \mathcal{A}_{\mathcal{D I R}}$ and $\left\|T_{A_{i}, B_{i}}\right\|_{\sigma}$ is the spectral operator norm of $T_{A_{i}, B_{i}}$.

Definition 2.10. Let $T_{A_{i}, B_{i}}: \mathcal{A}_{\mathcal{D I R}} \rightarrow \mathcal{B}_{\mathcal{D I R}}$ be a spectrally bounded operator, then $T_{B_{i}^{*}, A_{i}^{*}}: \mathcal{B}_{\mathcal{D I R}}^{*} \rightarrow \mathcal{A}_{\mathcal{D I} \mathcal{R}}^{*}$ defined by $T_{B_{i}^{*}, A_{i}^{*}} S=S \circ T_{A_{i}, B_{i}}$, for $S \in \mathcal{B}_{\mathcal{D} \mathcal{I R}}^{*}$ is said to be the spectral adjoint of $T_{A_{i}, B_{i}}$.

Definition 2.11. A linear mapping $T_{A_{i}, B_{i}}: \mathcal{A}_{\mathcal{D I R}} \rightarrow \mathcal{A}_{\mathcal{D I R}}$ is said to:

(i). compress left essential spectrum if $\sigma_{l e}\left(T_{A_{i}, B_{i}}(X)\right) \subseteq \sigma_{l e}(X), \forall X \in \mathcal{A}_{\mathcal{D I R}}$.

(ii). compress right essential spectrum if $\sigma_{r e}\left(T_{A_{i}, B_{i}}(X)\right) \subseteq \sigma_{r e}(X), \forall X \in \mathcal{A}_{\mathcal{D I R}}$.

(iii). compress semi-Fredholm spectrum if $\sigma_{s f}\left(T_{A_{i}, B_{i}}(X)\right) \subseteq \sigma_{e}(X), \forall X \in \mathcal{A}_{\mathcal{D I R}}$.

Definition 2.12. A linear mapping $T_{A_{i}, B_{i}}: \mathcal{A}_{\mathcal{D I R}} \rightarrow \mathcal{A}_{\mathcal{D I R}}$ is surjective up to compact operators if $\mathcal{A}_{\mathcal{D I R}}=\operatorname{Ran}\left(T_{A_{i}, B_{i}}\right)+$ $\mathcal{K}_{\mathcal{D I R}}$ and hence is called essentially spectrally bounded if there exists constant $M$ such that $r_{e}\left(T_{A_{i}, B_{i}}(X)\right) \leq M r_{e}(X)$, for all $X \in \mathcal{A}_{\mathcal{D I R} \text {. }}$

Definition 2.13. Let $\mathcal{A}_{\mathcal{D I R}}$ and $\mathcal{B}_{\mathcal{D I R}}$ be two dense irreducible subalgebras. Let $\mathcal{I} \subseteq \mathcal{A}_{\mathcal{D I R}}$ and $\mathcal{J} \subseteq \mathcal{B}_{\mathcal{D I R}}$ be closed proper ideals of dense irreducible subalgebras $\mathcal{A}_{\mathcal{D I R}}$ and $\mathcal{B}_{\mathcal{D I R}}$ respectively. A linear mapping $T_{A_{i}, B_{i}}: \mathcal{A}_{\mathcal{D I R}} \rightarrow \mathcal{B}_{\mathcal{D I R}}$ is surjective modulo $\mathcal{J}$ if for every $Y \in \mathcal{B}_{\mathcal{D I R}}$, there exists $X \in \mathcal{A}_{\mathcal{D I R}}$ such that $Y-T_{A_{i}, B_{i}}(X) \in \mathcal{J}$. We define the induced mapping $\widehat{T}_{A_{i}, B_{i}}: \mathcal{A}_{\mathcal{D I R}} / \mathcal{I} \rightarrow \mathcal{B}_{\mathcal{D I R}} / \mathcal{J}$ by $\widehat{T}_{A_{i}, B_{i}}(X+\mathcal{I})=T_{A_{i}, B_{i}}(X)+\mathcal{J}, X \in \mathcal{A}_{\mathcal{D I R}}$.

Definition 2.14. Let $T_{A_{i} B i}: \mathcal{A}_{\mathcal{D I R}} \rightarrow \mathcal{A}_{\mathcal{D I R}}$. The spectral operator norm is defined by

$$
\left\|T_{A_{i} B i}(X)\right\|_{\sigma}=\inf \left\{M \geq 0 r\left(T_{A_{i} B i}(X)\right) \leq M r(X), X \in \mathcal{A}_{\mathcal{D I R}}\right\} .
$$

Definition 2.15. A linear mapping $T_{A_{i}, B_{i}}: \mathcal{A}_{\mathcal{D I R}} \rightarrow \mathcal{B}_{\mathcal{D I R}}$ is said to be spectrally compact if $\overline{T_{A_{i}, B_{i}}\left(\mathcal{C}_{\mathcal{D I R}}\right)}$ is compact in $\mathcal{A}_{\mathcal{D I R}}$ where $\mathcal{C}_{\mathcal{D I R}}=\left\{X \in \mathcal{A}_{\mathcal{D I R}}: r(X) \leq 1\right\}$ and $\mathcal{C}_{\mathcal{D I R}} \subseteq \mathcal{A}_{\mathcal{D I R}}$

Definition 2.16. A representation $\Phi: \mathcal{A}_{\mathcal{D I R}} \rightarrow \mathcal{C}(\mathcal{E})$ is irreducible provided the $C^{*}$-subalgebra $\Phi\left(\mathcal{A}_{\mathcal{D I R}}\right)$ is irreducible in $\mathcal{C}(\mathcal{E})$, that is, there are no subalgebra for $\Phi\left(\mathcal{A}_{\mathcal{D I} \mathcal{R}}\right)$ that are reducing except for trivial ones $\{0\}$ and $A_{D I R}$. 


\section{Spectrally bounded compact elementary operators}

In this section, we characterize spectral boundedness of compact elementary operators on dense irreducible subalgebras of Banach algebra $\mathcal{A}$. We begin with the following proposition:

Proposition 3.1. Consider $L_{A}, R_{B}$ and $\delta_{A, B} \in \mathcal{C}(\mathcal{E})$. Let $\mathcal{A}_{\mathcal{D I R}}$ be a unital $C^{*}$-subalgebra of $\mathcal{A}$, then the following are equivalent:

(i). $L_{A}$ and $R_{B}$ are both spectrally bounded.

(ii). $\delta_{A, B}$ is spectrally bounded.

Proof. (i) $\Rightarrow$ (ii). From Definition 2.1, the left multiplication elementary operator is denoted by $L_{A}=A X$ for all $X \in \mathcal{A}_{\mathcal{D I R}}$ and $A$ fixed in $\mathcal{A}_{\mathcal{D I R}}$. It follows from [26] Proposition 2.1 that for some constant $M \geq 0, r(A X) \leq \operatorname{Mr}(X)$ holds hence $L_{A}$ is spectrally bounded. Consequently, $R_{A}=X A$ must be spectrally bounded. It is also known that inner derivation $\delta_{A} \in \operatorname{Rad}\left(\mathcal{A}_{\mathcal{D I R}}\right)$. So, if $A=B$ then $R_{B}$ is spectrally bounded and if $A-B=C$ then $R_{A-B}=R_{C}$ which is intuitively spectrally bounded. The generalized derivation can be expressed as:

$\delta_{A, B}(X)=L_{A}(X)-R_{B}(X)=R_{A-B}(X)+L_{A}(X)-R_{A}(X)=X(A-B)+A X-X A=X A-X B+A X-X A=A X-X B$.

Let a canonical homomorphism $\varphi: \mathcal{A}_{\mathcal{D I R}} \rightarrow \mathcal{A}_{\mathcal{D I R}} / \operatorname{Rad}\left(\mathcal{A}_{\mathcal{D I R}}\right)$ be a spectral isometry such that there exists a linear map $R: \mathcal{A}_{\mathcal{D I R}} \rightarrow \mathcal{A}_{\mathcal{D I R}}$ with $R \mathcal{A}_{\mathcal{D I R}} \subseteq \operatorname{Rad}\left(\mathcal{A}_{\mathcal{D I R}}\right)$. Therefore, if $N \in \mathcal{C}_{\mathcal{S B D}}(\mathcal{E})$ then $R+N \in \mathcal{C}_{\mathcal{S B D}}(\mathcal{E})$ and $r\left(\delta_{A, B}(X)\right)=$ $r\left(L_{A}(X)-R_{B}(X)\right)=r\left(R_{A-B}(X)+L_{A}(X)-R_{A}(X)\right)$ holds. Hence, $r\left(\delta_{A, B}(X)\right) \leq M r(X)$ must be spectrally bounded.

(ii) $\Rightarrow$ (i). We suppose that $\delta_{A, B}$ is spectrally bounded, then $r\left(\delta_{A, B}(X)\right) \leq M r(X)$ for some constant $M \geq 0$. We need to prove that $L_{A}$ and $R_{B}$ are both spectrally bounded, that is, $r\left(L_{A}(X) \leq M r(X)\right.$ and $r\left(R_{B}(X) \leq M r(X)\right.$ for some constant $M \geq 0$. Let a canonical homomorphism $\varphi: \mathcal{A}_{\mathcal{D I R}} \rightarrow \mathcal{A}_{\mathcal{D I R}} / \operatorname{Rad}\left(\mathcal{A}_{\mathcal{D I R}}\right)$ be a spectral isometry such that $B$ is central modulo the $\operatorname{Rad}\left(\mathcal{A}_{\mathcal{D I R}}\right)$, thus

$$
\begin{aligned}
r\left(\delta_{A, B}\right) & =r(A X-X B)=r(\varphi(A) \varphi(X)-\varphi(X) \varphi(B))=r((\varphi(A)-\varphi(B)) \varphi(X))=r(\varphi(X)(\varphi(A)-\varphi(B))) \\
& =r(\varphi(B) \varphi(X)-\varphi(X) \varphi(A))=r(B X-X A), X \in \mathcal{A}_{\mathcal{D I R}} .
\end{aligned}
$$

Therefore, $r\left(\delta_{B, A}(X)\right)=r\left(\delta_{A, B}(X)\right) \leq M r(X)$ is spectrally bounded. Now, suppose that $R_{B}$ is not spectrally bounded. Then there exists an irreducible representation $\Phi$ of $\mathcal{A}_{\mathcal{D I R}}$ such that $\Phi(B) \neq I_{\mathbb{C}}$. Let a vector $v$ be in the representation space such that $\{v, \Phi(B) v\}$ is linearly independent. Since $\Phi$ is a continuous representation of $\mathcal{A}_{\mathcal{D I R}}$ on a Banach space $\Omega$, then from Jacob density theorem, there exists $X, E \in \mathcal{A}_{\mathcal{D I R}}$ such that $\Phi(X) v=0, \Phi(X) \Phi(B) v=0, \Phi(E) \Phi(B) v=\Phi(B) v$, $\Phi(E) v=-n v$, whenever $n \in \mathbb{N}$. Furthermore, from Sinclair's theorem, we assume that $E$ is invertible such that

$$
\left(\Phi(A) \Phi(E) \Phi(X) \Phi(E)^{-1}-\Phi(E) \Phi(X) \Phi(E)^{-1} \Phi(B)\right) v=n v .
$$

Hence, $r\left(A E X E^{-1}-E X E^{-1} B\right) \geq n$ which contradicts the hypothesis that $r\left(A E X E^{-1}-E X E^{-1} B\right) \leq M r\left(E X E^{-1}\right)=$ $M r(X)$ and for some $M \geq 0$. Thus, $R_{B}$ must be spectrally bounded. Analogously, $L_{A}$ is also spectrally bounded. Therefore, $L_{A}$ and $R_{B}$ are both spectrally bounded.

Proposition 3.2. Let $L_{A}: \mathcal{A}_{\mathcal{D I R}} \rightarrow \mathcal{A}_{\mathcal{D I R}}$ be compact and spectrally bounded, then $L_{A}$ maps into the radical of $\mathcal{A}_{\mathcal{D I R}}$ if and only if $\mathcal{A}_{\mathcal{D I R}}$ is a $C^{*}$-subalgebra of $\mathcal{A}$ with identity.

Proof. Let $L_{A}$ be compact, spectrally bounded and maps into radical of $\mathcal{A}_{\mathcal{D I R}}$. We prove that $\mathcal{A}_{\mathcal{D I R}}$ is a $C^{*}$-subalgebra of $A$ with identity. Let $X \in \mathcal{A}_{\mathcal{D I R}}$ such that $(I-X)^{-1}=\sum_{n=0}^{\infty} X^{n}$ and $\|X\|=\gamma<1$ with $C_{i}=\sum_{n=0}^{i} X^{i}$. Indeed, $\left(C_{i}\right)$ is a cauchy sequence converging to some element $E=\sum_{n=0}^{\infty} X^{n}$ since for $i<j$ it holds that

$$
\left\|C_{i}-C_{j}\right\| \leq \sum_{n=i+1}^{j}\|X\|^{n} \leq \frac{\gamma^{i+1}}{1-\gamma}
$$

Now, $X C_{i}=C_{i+1}-I$ and $E(I-X)=(I-X) E=I$ and hence $\mathcal{A}_{\mathcal{D I R}}$ is a $C^{*}$-subalgebra of $\mathcal{A}$ with identity.

Conversely, let $\mathcal{A}_{\mathcal{D I R}}$ be a $C^{*}$-subalgebra with identity. We prove that $L_{A}$ is compact, spectrally bounded and maps into radical of $\mathcal{A}_{\mathcal{D I R}}$. It follows from Proposition 3.1, $L_{A}$ is compact and spectrally bounded such that $r(A X) \leq \operatorname{Mr}(X)$ for some constant $M \geq 0$. In addition, to show that $L_{A}$ maps into the $\operatorname{rad}\left(A_{D I R}\right)$ then we need to prove that intersection of all maximal left ideals must be invertible in $\mathcal{A}_{\mathcal{D I R}}$ and any invertible element of $A_{D I R}$ is contained in the intersection of all left maximal ideal. We begin with showing that the intersection of all maximal left ideals must be invertible in $\mathcal{A}_{\mathcal{D I R}}$. Suppose that $X$ is in the intersection of all maximal left ideals of $\mathcal{A}_{\mathcal{D I R}}$. If $L=I-A X$ is not invertible then $\mathcal{A}_{\mathcal{D I R}} L$ is a 
left ideal. This is because every left ideal of $\mathcal{A}_{\mathcal{D I} \mathcal{R}}$ is contained in a maximal left ideal. Now, $A X \in \mathcal{L}_{0}$ and $I-A X \in \mathcal{L}_{0}$ which implies that $I \in \mathcal{L}_{0}$ where $\mathcal{L}_{0}$ is some left ideal. Therefore, $\mathcal{L}_{0}=\mathcal{A}_{\mathcal{D I R}}$ which is a contradiction. Next, we show that any invertible element of $A_{D I R}$ is contained in the intersection of all left maximal ideal. Suppose that $I-A X$ is invertible

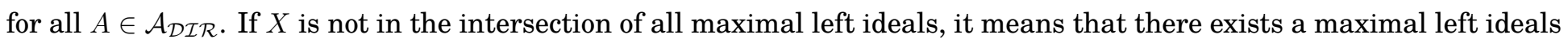
$\mathcal{L}_{0}$ such that $X \notin \mathcal{L}_{0}$. Therefore, $\mathcal{L}_{0}+\mathcal{A}_{\mathcal{D I R}} L=\mathcal{A}_{\mathcal{D I R}}$ and $I-A X \in \mathcal{L}_{0}$, but this gives a contradiction because $I \in \mathcal{L}_{0}$. Hence, $L_{A}$ maps into the radical of $\mathcal{A}_{\mathcal{D I} \mathcal{R}}$.

Lemma 3.1. Let $R_{B}: \mathcal{A}_{\mathcal{D I R}} \rightarrow \mathcal{A}_{\mathcal{D I R}}$ be compact and spectrally bounded, then $R_{B}$ maps into the radical of $\mathcal{A}_{\mathcal{D I R}}$ if and only if $\mathcal{A}_{\mathcal{D I R}}$ is a $C^{*}$-subalgebra with identity.

Proof. Let $R_{B}$ be compact, spectrally bounded and maps into radical of $\mathcal{A}_{\mathcal{D I R}}$. We prove that $\mathcal{A}_{\mathcal{D I R}}$ is a $C^{*}$-subalgebra of $A$ with identity. It follows analogously from Proposition 3.2 that $\mathcal{A}_{\mathcal{D I R}}$ is $C^{*}$-subalgebra of $\mathcal{A}$ with identity.

Conversely, let $\mathcal{A}_{\mathcal{D I R}}$ be a $C^{*}$-subalgebra with identity. We prove that $R_{B}$ is compact, spectrally bounded and maps into radical of $\mathcal{A}_{\mathcal{D I R}}$. It follows from Proposition 3.1, $R_{B}$ is compact and spectrally bounded such that $r(X B) \leq \operatorname{Mr}(X)$ for some constant $M \geq 0$. In addition, to show that $R_{B}$ maps into the $\operatorname{rad}\left(A_{D I R}\right)$ then we need to prove that intersection of all maximal right ideals must be invertible in $\mathcal{A}_{\mathcal{D I R}}$ and any invertible element of $A_{D I R}$ is contained in the intersection of all right maximal ideal. We begin with showing that the intersection of all maximal right ideals must be invertible in $\mathcal{A}_{\mathcal{D I R}}$. Suppose that $X$ is in the intersection of all maximal right ideals of $\mathcal{A}_{\mathcal{D I R}}$. If $R=I-X B$ is not invertible then $R \mathcal{A}_{\mathcal{D I R}}$ is a right ideal. This is because every right ideal of $\mathcal{A}_{\mathcal{D I R}}$ is contained in a maximal right ideal. Now, $X B \in \mathcal{R}_{0}$ and $I-X B \in \mathcal{R}_{0}$ which implies that $I \in \mathcal{R}_{0}$ where $\mathcal{R}_{0}$ is some right ideal. Therefore, $\mathcal{R}_{0}=\mathcal{A}_{\mathcal{D I R}}$ which is a contradiction. Next, we show that any invertible element of $A_{D I R}$ is contained in the intersection of all right maximal ideal. Suppose that $I-X B$ is invertible for all $B \in \mathcal{A}_{\mathcal{D I R}}$. If $X$ is not in the intersection of all maximal right ideals, it means that there exists a maximal right ideals $\mathcal{R}_{0}$ such that $X \notin \mathcal{R}_{0}$. Therefore, $R \mathcal{A}_{\mathcal{D I R}}+\mathcal{R}_{0}=\mathcal{A}_{\mathcal{D I R}}$ and $I-X B \in \mathcal{R}_{0}$, but this gives a contradiction because $I \in \mathcal{R}_{0}$. Thus, $R_{B}$ maps into the radical of $\mathcal{A}_{\mathcal{D I R}}$.

Theorem 3.1. Let $M_{A, B}: \mathcal{A}_{\mathcal{D I R}} \rightarrow \mathcal{A}_{\mathcal{D I R}}$ be compact and spectrally bounded, then $M_{A, B}$ maps into the radical of $\mathcal{A}_{\mathcal{D I R}}$ if and only if $\mathcal{A}_{\mathcal{D I R}}$ is a $C^{*}$-subalgebra of $\mathcal{A}$ with identity.

Proof. Suppose that $M_{A, B}$ is compact, spectrally bounded and maps into the radical of $\mathcal{A}_{\mathcal{D I R}}$. We need to show that $\mathcal{A}_{\mathcal{D I R}}$ is a $C^{*}$-subalgebra of $\mathcal{A}$ with identity. Indeed, this follows analogously from Proposition 3.2 that $\mathcal{A}_{\mathcal{D I R}}$ is $C^{*}$-subalgebra of $\mathcal{A}$ with identity.

Conversely, let $\mathcal{A}_{\mathcal{D I R}}$ be a $C^{*}$-subalgebra of $\mathcal{A}$ with identity. We need to show that $M_{A, B}$ is compact, spectrally bounded and maps into the radical of $\mathcal{A}_{\mathcal{D I R}}$. From Definition 2.1, the basic elementary operator is denoted by $M_{A, B}(X)=A X B$ for all $X \in \mathcal{A}_{\mathcal{D I R}}$ and $A, B$ fixed in $\mathcal{A}_{\mathcal{D I R}}$. Let $X$ be a projection in $\mathcal{A}_{\mathcal{D I R}}$, that is, $X^{2}=X$ such that $L_{A} R_{B}=A X \cdot X B=$ $A X^{2} B=A X B=B A X$. Since $r(X)=\|X\|$ and $r(A X B)=r(B A X)$, it follows from Proposition 2.4 of [18] that

$$
r\left(M_{A, B}(X)\right)=r(A X B)=r(B A X) \leq\|B\|_{\sigma} r(A X) \leq\|B\|_{\sigma}\|A\|_{\sigma} r(X)=M r(X) .
$$

Hence, $r\left(M_{A X B}\right) \leq M r(X)$ is spectrally bounded for some $M \geq 0$. Now, to prove that $M_{A, B}(X)$ maps into the radical, we need to show that the intersection of all maximal left (right) ideals are invertible in $\mathcal{A}_{\mathcal{D I R}}$ and any invertible element of $\mathcal{A}_{\mathcal{D I R}}$ is contained in the intersection of all maximal left (right) ideal. Let $X \in A_{D I R}$ be in the intersection of all maximal left (right) ideal of $\mathcal{A}_{\mathcal{D I R}}$ then $I-A X B$ is invertible in $\mathcal{A}_{\mathcal{D I R}}$ for any $A, B$ fixed in $\mathcal{A}_{\mathcal{D I R}}$. This is because by Proposition 3.2, $I-A X B$ is left invertible by letting $B=I$ and by Lemma 3.1, $I-A X B$ is right invertible by letting $A=I$ hence $A X B$ must be invertible in $\mathcal{A}_{\mathcal{D I R}}$. Finally, let $E \in \mathcal{A}_{\mathcal{D I R}}$ such that $E(I-A X B)=(I-A X B) E=I$ holds. Since $A X \in \mathcal{L}_{0}$ and $X B \in \mathcal{R}_{0}$, it implies that $A X B$ is in the maximal ideal $\mathcal{M}_{0}$ since $E(I-A X B)=(I-A X B) E=I$. Therefore, $E=I+E(A X B)$ is left invertible and $E$ is maximal ideal. Thus, $I-A X B$ and $A X B$ are contained in the maximal ideal $\mathcal{M}_{0}$. Therefore $M_{A, B}$ maps into the radical of $\mathcal{A}_{\mathcal{D I R}}$.

Corollary 3.1. Every elementary operator $T_{A_{i}, B_{i}}: \mathcal{A}_{\mathcal{D I R}} \rightarrow \mathcal{A}_{\mathcal{D I R}}$ defined by $T_{A_{i}, B_{i}}(X)=\sum_{i=1}^{n} A_{i} X B_{i}$ is spectrally bounded.

Proof. From Theorem 3.1, $M_{A, B}(X)$ is spectrally bounded and since $T_{A_{i}, B_{i}}$ is the sum of basic elementary operators, it follows immediately that $T_{A_{i}, B_{i}}$ is spectrally bounded, that is, $r\left(T_{A_{i}, B_{i}}(X)\right) \leq \operatorname{Mr}(X)$.

Remark 3.1. If $\left(L_{A}-R_{A}\right) \mathcal{A}_{\mathcal{D I R}} \subsetneq \operatorname{Rad}\left(\mathcal{A}_{\mathcal{D I R}}\right)$ that is $A$ is not central modulo $\operatorname{Rad}\left(\mathcal{A}_{\mathcal{D I R}}\right)$ then $R_{A}$ and hence $L_{A}$ cannot be spectrally bounded.

Theorem 3.2. Let $T_{A_{i}, B_{i}} \in \mathcal{C}(\mathcal{E})$ be spectrally compact. Then $T_{A_{i}, B_{i}}$ is a compact operator if and only if it is spectrally bounded. 
Proof. Suppose that $T_{A_{i}, B_{i}}$ is spectrally bounded. We prove that $T_{A_{i}, B_{i}}$ is a compact operator. Let

$$
\mathcal{C}_{\mathcal{D I R}}=\left\{X \in \mathcal{A}_{\mathcal{D I R}}: r(X) \leq 1\right\}
$$

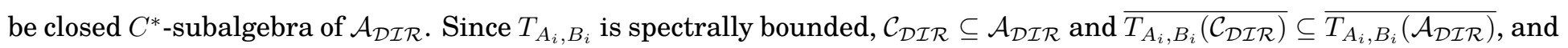
thus $\overline{T_{A_{i}, B_{i}} \mathcal{C}_{\mathcal{D I R}}}$ is spectrally compact and hence compact.

Conversely, let $T_{A_{i}, B_{i}}$ be compact. We show that $T_{A_{i}, B_{i}}$ is spectrally bounded. We prove by contradiction. Suppose that $T_{A_{i}, B_{i}}$ is not spectrally bounded. We know that $T_{A_{i}, B_{i}}$ attains its spectral operator norm if for some positive number $M \geq 0$ then $\left\|T_{A_{i}, B_{i}}(X)\right\|_{\sigma} \leq M$, for all $X \in \mathcal{A}_{\mathcal{D I R} \text {. If }} r(X)>0$ for any $X \in \mathcal{A}_{\mathcal{D I R}},\left\|T_{A_{i}, B_{i}}\left(\frac{X}{r(X)}\right)\right\|_{\sigma} \leq M$ and $r\left(T_{A_{i}, B_{i}}(X)\right) \leq M r(X)$ is spectrally bounded. Choose $\varepsilon>0$ arbitrarily such that $r(X)=0$, then

$$
r\left(\frac{X}{\frac{\varepsilon}{M}}\right)=0 \Rightarrow\left\|T_{A_{i}, B_{i}}\left(\frac{X}{\frac{\xi}{M}}\right)\right\|_{\sigma} \leq M \quad \text { and } \quad\left\|T_{A_{i}, B_{i}}(X)\right\|_{\sigma} \leq M \cdot \frac{\varepsilon}{M}=\varepsilon .
$$

Therefore, $r\left(T_{A_{i}, B_{i}} X\right) \leq\left\|T_{A_{i}, B_{i}}(X)\right\|_{\sigma}=0$ since $r(X)=0$. Hence, $r\left(T_{A_{i}, B_{i}}(X)\right) \leq M r(X)$ for all $X \in \mathcal{A}_{\mathcal{D I R}}$, which is a contradiction. Hence, by Corollary 3.1, $T_{A_{i}, B_{i}}$ must be spectrally bounded.

Example 3.1. Suppose that $\mathcal{A}_{\mathcal{D I R}}$ is dense irreducible commutative $C^{*}$-subalgebra and $\mathbb{M}_{2}(\mathbb{C})$ is $C^{*}$-subalgebra of Banach algebra $\mathcal{A}$. Let $\Phi$ be an irreducible representation of $\mathcal{A}_{\mathcal{D I R}}$ on $\mathbb{C}$ and $\Psi$ be an unbounded character on $\mathcal{A}_{\mathcal{D I R}}$. Then, the operator $T_{A_{i}, B_{i}}: \mathcal{A}_{\mathcal{D I R}} \rightarrow \mathbb{M}_{2}(\mathbb{C})$ having the representation

$$
T_{A_{i}, B_{i}}(X)=\left(\begin{array}{cc}
\Phi(X) & \Psi(X) \\
0 & \Phi(X)
\end{array}\right)
$$

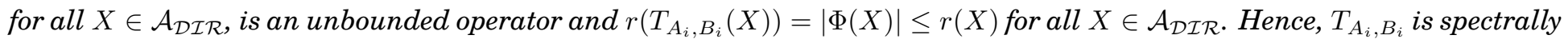
bounded elementary but not compact; otherwise, by Theorem 3.2, $T_{A_{i}, B_{i}}$ should be spectrally bounded and compact operator.

Theorem 3.3. Every compact and spectrally bounded $C^{*}$-subalgebra $\mathcal{C}_{\mathcal{D I R}}$ is complete.

Proof. Let $X_{n}$ be spectrally bounded sequence in $\mathcal{A}_{\mathcal{D I R}}$ such that $r\left(X_{n}\right) \leq M$ for some positive number $M \geq 0$ and $n \in \mathbb{N}$. If $\mathcal{C}_{\mathcal{D I R}}=\left\{X \in \mathcal{A}_{\mathcal{D I R}}: r(X) \leq M\right\}$ is a spectrally bounded $C^{*}$-subalgebra of $\mathcal{A}_{\mathcal{D I R}}$ then $\overline{T_{A_{i}, B_{i}}\left(\mathcal{C}_{\mathcal{D I R}}\right)}$ is compact and $\left(T_{A_{i}, B_{i}}\left(X_{n}\right)\right)$ is a sequence in the compact subalgebra. To show that $C_{D I R}$ is complete, we prove that every converging sequence in the compact subalgebra $\overline{T_{A_{i}, B_{i}}\left(\mathcal{C}_{\mathcal{D I R}}\right)}$ has a convergent subsequence. We apply the approximation property from Definition 2.5 for compact $C^{*}$-subalgebra $\mathcal{C}_{\mathcal{D I R}}$ of $\mathcal{A}_{\mathcal{D I R}}$. Let $Y_{n}$ be a sequence in $\overline{T_{A_{i}, B_{i}}\left(\mathcal{C}_{\mathcal{D I R}}\right)}$ and for all $n \in \mathbb{N}$ then $\left\|Y_{n}-T_{A_{i}, B_{i}}\left(X_{n}\right)\right\|<\frac{1}{n}, X_{n} \in \mathcal{C}_{\mathcal{D I R}}$. Since the sequence $X_{n}$ is spectrally bounded, then there exists subsequence $T_{A_{i}, B_{i}}\left(X_{n_{k}}\right)$ of $T_{A_{i}, B_{i}}\left(X_{n}\right)$ such that $\lim _{k \rightarrow \infty} T_{A_{i}, B_{i}}\left(X_{n_{k}}\right) \rightarrow Y$, for every $Y \in \mathcal{B}_{\mathcal{D I R} \mathcal{R}}$. Let an arbitrary $\varepsilon>0$ be given. There are $K_{1}, K_{2} \in \mathbb{N}$ such that $\frac{1}{n_{k}}<\frac{\varepsilon}{2} \forall k \geq K_{1}$ and $\left\|T_{A_{i}, B_{i}}\left(X_{n_{k}}\right)-Y\right\|<\frac{\varepsilon}{2}$ for every $k \geq K_{2}$. Finally, suppose that $k \geq \max \left\{K_{1}, K_{2}\right\}$ then

$$
\left\|Y_{n_{k}}-Y\right\|=\left\|Y_{n_{k}}-T_{A_{i}, B_{i}}\left(X_{n_{k}}\right)+T_{A_{i}, B_{i}}\left(X_{n_{k}}\right)-Y\right\| \leq\left\|Y_{n_{k}}-T_{A_{i}, B_{i}}\left(X_{n_{k}}\right)\right\|+\left\|T_{A_{i}, B_{i}}\left(X_{n_{k}}\right)-Y\right\|<\frac{\varepsilon}{2}+\frac{\varepsilon}{2}=\varepsilon .
$$

Therefore, the subsequence $Y_{n_{k}} \rightarrow Y$ converges.

Theorem 3.4. Let $\mathcal{C}_{\mathcal{D I R}}$ be a closed $C^{*}$-subalgebra of $\mathcal{A}_{\mathcal{D I R}}$. If $T_{A_{i}, B_{i}}: \mathcal{C}_{\mathcal{D I R}} \rightarrow \mathcal{B}_{\mathcal{D I R}}$ is a spectrally bounded compact elementary operator, then $T_{A_{i}, B_{i}}$ has a spectrally bounded compact elementary operator extension $\widetilde{T}_{A_{i}, B_{i}}: \mathcal{A}_{\mathcal{D I R}} \rightarrow \mathcal{B}_{\mathcal{D I R}}$, where both $T_{A_{i}, B_{i}}$ and $\widetilde{T}_{A_{i}, B_{i}}$ acts on sequences of maps with a unique point of convergence.

Proof. By Theorem 3.2, $T_{A_{i}, B_{i}}$ is spectrally bounded compact elementary operator and the extension $\widetilde{T}_{A_{i}, B_{i}}: \mathcal{A}_{\mathcal{D I R}} \rightarrow \mathcal{B}_{\mathcal{D I R}}$ is guaranteed by spectral extension property. Now, we only check the uniqueness of the point of convergence of $\widetilde{T}_{A_{i}, B_{i}}$. Let $X_{k}$ be a spectrally bounded sequence in $\mathcal{A}_{\mathcal{D I R}}$ such that $r\left(X_{k}\right) \leq M$, for some constant $M \geq 0$ and for all $k \in \mathbb{N}$. Suppose we consider the set $U=\{\lambda \in \mathbb{C}:|\lambda|<M\}$ such that $\sigma\left(X_{k}\right) \subseteq U$, for all $k \in \mathbb{N}$. We note from Theorem 3.3 that $\mathcal{C}_{\mathcal{D I R}}$ is compact and complete. Hence, any $X_{1} \in \overline{\mathcal{C}_{\mathcal{D I R}}}$ has a sequence $X_{1_{k}} \in \mathcal{C}_{\mathcal{D I R}}$ converging to $X_{1}$ i.e $X_{1_{k}} \rightarrow X_{1}$. So applying upper semi-continuous property of the spectrum, we have $\left\|X_{1}-Z\right\|<\gamma_{1}$ for $0<\gamma_{1}<1$ implies $\sigma(Z) \subseteq U$ hence $r(Z) \leq M$. Choose $0<\varrho_{1}<\gamma_{1}$, then there exists $k_{1} \in \mathbb{N}$ such that $\left\|X_{1_{k}}-X_{1}\right\|<\varrho_{1}<1$ for every $k \geq k_{1}$. Hence $\sigma\left(X_{1_{k}}\right) \subseteq U$ for every $k \geq k_{1}$. Again, we choose $X_{2} \in \overline{\mathcal{C}_{\mathcal{D I R}}}$ such that the sequence $X_{2_{k}} \in \mathcal{C}_{\mathcal{D I R}}$ converges to $X_{2}$ i.e $X_{2_{k}} \rightarrow X_{2}$. Again applying upper semi-continuous property of the spectrum, we have $\left\|X_{2}-Z\right\|<\gamma_{2}$ for $0<\gamma_{2}<\frac{1}{2}$ implies $\sigma(Z) \subseteq U$ hence $r(Z) \leq M$. If $0<\varrho_{2}<\gamma_{2}$, then there exists $k_{2} \in \mathbb{N}$ such that $\left\|X_{2_{k}}-X_{2}\right\|<\varrho_{2}<\frac{1}{2}$ for every $k \geq k_{2}$. Hence $\sigma\left(X_{2_{k}}\right) \subseteq U$ for every $k \geq k_{2}$. By induction, the sequence $X_{i_{k}}$ converges $X_{i}$ such that $X_{k_{i}}-X_{i_{k_{i}}} \rightarrow 0$ and $\sigma\left(X_{i_{k}}\right) \subseteq U$. Using completeness property of $T_{A_{i}, B_{i}}$ there exists a converging subsequence $X_{i_{i}}$ such that $T_{A_{i}, B_{i}}\left(X_{i_{k_{i}}}\right) \rightarrow Y_{0}$ for some $Y_{0} \in \mathcal{B}_{\mathcal{D I R}}$ as $i \rightarrow \infty$ and applying continuity of $\widetilde{T}_{A_{i}, B_{i}}$, we have $\widetilde{T}_{A_{i}, B_{i}}\left(X_{k_{i}}\right)-\widetilde{T}_{A_{i}, B_{i}}\left(X_{i_{k_{i}}}\right)=\widetilde{T}_{A_{i}, B_{i}}\left(X_{k_{i}}-X_{i_{k_{i}}}\right) \rightarrow 0$. Therefore, $\widetilde{T}_{A_{i}, B_{i}}\left(X_{k_{i}}\right) \rightarrow Y_{0}$ and $X_{k}$ has a unique point of convergence $Y_{0}$ for both $T_{A_{i}, B_{i}}$ and $\widetilde{T}_{A_{i}, B_{i}}$. 
Corollary 3.2. Let $T_{A_{i}, B_{i}}: \mathcal{A}_{\mathcal{D I R}} \rightarrow \mathcal{B}_{\mathcal{D I R}}$ be a spectrally bounded compact elementary operator. Then the spectral adjoint $T_{B_{i}^{*}, A_{i}^{*}}: \mathcal{B}_{\mathcal{D I R}}^{*} \rightarrow \mathcal{A}_{\mathcal{D I R}}^{*}$ is totally bounded if and only if $\mathcal{B}_{\mathcal{D I R}}^{*}$ is a spectrally bounded $C^{*}$-subalgebra of $\mathcal{A}$.

Proof. Suppose that $T_{B_{i}^{*}, A_{i}^{*}}$ is totally bounded. We show that $\mathcal{B}_{\mathcal{D} \mathcal{L} \mathcal{R}}^{*}$ is a spectrally bounded $C^{*}$-subalgebra of $\mathcal{A}$. Let $\left.\left\{S_{a}\right\}\right|_{a \in \mathbb{N}}$ be a cauchy sequence in $\mathcal{B}_{\mathcal{D} \mathcal{I R}}^{*}$. Then for any $\varepsilon>0$ and $a, b \geq n \in \mathbb{N}$, we have

$$
\left|S_{a}(X)-S_{b}(X)\right|=r\left(\left(S_{a}-S_{b}\right) X\right) \leq\left\|S_{a}-S_{b}\right\|_{\sigma} r(X)<\varepsilon r(X), \text { for all } X \in \mathcal{A}_{\mathcal{D I R}}
$$

In particular, the $\left\|T_{B_{i}^{*}, A_{i}^{*}}\right\|_{\sigma} \leq\left\|T_{A_{i}, B_{i}}\right\|_{\sigma}$ and $T_{B_{i}^{*}, A_{i}^{*}} S=S \circ T_{A_{i}, B_{i}}$, for all $S \in \mathcal{B}_{\mathcal{D} \mathcal{I R}}^{*}$ and thus $T_{B_{i}^{*}, A_{i}^{*}}$ is spectrally bounded. Now,

$$
r\left(T_{B_{i}^{*}, A_{i}^{*}} S\right)=\left\|S \circ T_{A_{i}, B_{i}}\right\| \leq\|S\|_{\sigma}\left\|T_{A_{i}, B_{i}}\right\|_{\sigma}=\left\|T_{A_{i}, B_{i}}\right\|_{\sigma} r(S) \leq M r(S), \text { for all } S \in \mathcal{B}_{\mathcal{D I R}}^{*} .
$$

Hence, $\mathcal{B}_{\mathcal{D} \mathcal{L}}^{*}$ is a spectrally bounded $C^{*}$-subalgebra of $\mathcal{A}$.

Conversely, let $\mathcal{B}_{\mathcal{D} \mathcal{L}}^{*}$ be a spectrally bounded $C^{*}$-subalgebra of $\mathcal{A}$. We show that $T_{B_{i}^{*}, A_{i}^{*}}$ is totally bounded. So, we consider $\mathcal{C}_{\mathcal{D I R}}^{*}$ a closed $C^{*}$-subalgebra of $\mathcal{B}_{\mathcal{D} \mathcal{I R}}^{*}$ such that for some constant $M>0$ and for every $S \in \mathcal{C}_{\mathcal{D I R}}^{*}$ then

$$
\|S\|_{\sigma}=r(S) \leq M .
$$

This shows that $S$ is spectrally bounded. From Theorem 3.3, $T_{A_{i}, B_{i}}$ is a spectrally bounded compact elementary operator so that $\overline{T_{A_{i}, B_{i}}\left(\mathcal{C}_{\mathcal{D I R}}\right)}$ is compact and complete. Thus, given $\varepsilon>0$, then the sequence $X_{1}, \ldots, X_{n} \in \mathcal{C}_{\mathcal{D I R}}$ is totally bounded for $k \in\{1, \ldots, n\}$ and $\left\|T_{A_{i}, B_{i}}(X)-T_{A_{i}, B_{i}}\left(X_{k}\right)\right\|<\frac{\varepsilon}{3 M}$. Hence, $\mathcal{C}_{\mathcal{D I R}}$ is totally bounded. Now, we check if $\mathcal{C}_{\mathcal{D} \mathcal{L}}^{*}$ is a totally bounded $C^{*}$-subalgebra. Let an irreducible representation $\Phi: \mathcal{B}_{\mathcal{D I R}}^{*} \rightarrow \mathbb{C}^{n}$ be defined by

$$
\Phi(S)=\left(S\left(T_{A_{i}, B_{i}}\left(X_{1}\right)\right)\right), \ldots,\left(S\left(T_{A_{i}, B_{i}}\left(X_{n}\right)\right)\right) \text {, for every } S \in \mathcal{B}_{\mathcal{D} \mathcal{I R}}^{*} .
$$

By Equation (1), $S \in \mathcal{B}_{\mathcal{D} \mathcal{L R}}^{*}$ is spectrally bounded and $T_{A_{i}, B_{i}}$ is compact operator. It follows that $\Phi$ is a compact operator and $\overline{\Phi\left(\mathcal{C}_{\mathcal{D I R}}^{*}\right)}$ is also compact $C^{*}$-subalgebra. To see that $\Phi\left(\mathcal{C}_{\mathcal{D} \mathcal{I R}}^{*}\right)$ is totally bounded, consider the sequence $S_{1}, \ldots, S_{a} \in \mathcal{C}_{\mathcal{D} \mathcal{I R}}^{*}$ such that for any $\varepsilon>0$ then there exists $m \in\{1,2, \ldots, a\}$ with $\left\|\Phi S-\Phi S_{m}\right\|<\frac{\varepsilon}{3}$. Thus, for every $S \in \mathcal{C}_{\mathcal{D I} \mathcal{R}}^{*}$ then there exists $m \in\{1,2, \ldots, a\}$ such that

$$
\left|S\left(T_{A_{i}, B_{i}}\left(X_{k}\right)\right)-S_{m}\left(T_{A_{i}, B_{i}}\left(X_{k}\right)\right)\right|^{2} \leq \sum_{b=1}^{n}\left|S\left(T_{A_{i}, B_{i}}\left(X_{b}\right)\right)-S_{m}\left(T_{A_{i}, B_{i}}\left(X_{b}\right)\right)\right|^{2}=\left\|\Phi\left(S-S_{m}\right)\right\|^{2}<\frac{\varepsilon^{2}}{3^{2}},
$$

for every $k \in\{1, \ldots, n\}$. Indeed, for any $X \in \mathcal{C}_{\mathcal{D I R}}$ and $S \in \mathcal{C}_{\mathcal{D I}}^{*}$, then $\left\|T_{A_{i}, B_{i}}(X)-T_{A_{i}, B_{i}}\left(X_{k}\right)\right\|<\frac{\varepsilon}{3 M}$ for $k \in\{1, \ldots, n\}$ and $\left\|\Phi S-\Phi S_{m}\right\|<\frac{\varepsilon}{3}$ for $m \in\{1,2, \ldots, a\}$. Thus, $\|S\| \leq\|S\|_{\sigma} \leq M$ holds for every $S \in \mathcal{C}_{\mathcal{D} \mathcal{I R}}^{*}$. Consequently, we have

$$
\begin{aligned}
\left|S\left(T_{A_{i}, B_{i}}(X)\right)-S_{m}\left(T_{A_{i}, B_{i}}(X)\right)\right|= & \mid S\left(T_{A_{i}, B_{i}}(X)\right)-S\left(T_{A_{i}, B_{i}}\left(X_{k}\right)\right)+S\left(T_{A_{i}, B_{i}}\left(X_{k}\right)\right)-S_{m}\left(T_{A_{i}, B_{i}}\left(X_{k}\right)\right) \\
& +S_{m}\left(T_{A_{i}, B_{i}}\left(X_{k}\right)\right)-S_{m}\left(T_{A_{i}, B_{i}}(X)\right) \mid \\
\leq & \left|S\left(T_{A_{i}, B_{i}}(X)\right)-S\left(T_{A_{i}, B_{i}}\left(X_{k}\right)\right)\right|+\left|S\left(T_{A_{i}, B_{i}}\left(X_{k}\right)\right)-S_{m}\left(T_{A_{i}, B_{i}}\left(X_{k}\right)\right)\right| \\
& +\left|S_{m}\left(T_{A_{i}, B_{i}}\left(X_{k}\right)\right)-S_{m}\left(T_{A_{i}, B_{i}}(X)\right)\right| \\
\leq & \left.\|S\| \cdot\left\|T_{A_{i}, B_{i}}(X)-T_{A_{i}, B_{i}}\left(X_{k}\right)\right\|+\| \Phi S-\Phi S_{m}\right)\|+\| S\|\cdot\| T_{A_{i}, B_{i}}\left(X_{k}\right)-T_{A_{i}, B_{i}}(X) \| \\
< & M \cdot \frac{\varepsilon}{3 M}+\frac{\varepsilon}{3}+M \cdot \frac{\varepsilon}{3 M}=\varepsilon .
\end{aligned}
$$

Now,

$$
\begin{aligned}
\left\|T_{B_{i}^{*}, A_{i}^{*}}(S)-T_{B_{i}^{*}, A_{i}^{*}}\left(S_{m}\right)\right\|_{\sigma} & =\sup \left\{r\left(\left(T_{B_{i}^{*}, A_{i}^{*}}(S)-T_{B_{i}^{*}, A_{i}^{*}}\left(S_{m}\right)\right)(X)\right): r(X) \leq 1\right\} \\
& =\sup \left\{\left|\left(T_{B_{i}^{*}, A_{i}^{*}}(S)-T_{B_{i}^{*}, A_{i}^{*}}\left(S_{m}\right)\right)(X)\right|: r(X) \leq 1\right\} \\
& =\sup \left\{\left|S\left(T_{A_{i}, B_{i}}(X)\right)-S_{m}\left(T_{A_{i}, B_{i}}(X)\right)\right|: r(X) \leq 1\right\} \\
& \leq \varepsilon .
\end{aligned}
$$

Hence, for every $S \in \mathcal{C}_{\mathcal{D} \mathcal{I} \mathcal{R}}^{*},\left\|T_{B_{i}^{*}, A_{i}^{*}}(S)-T_{B_{i}^{*}, A_{i}^{*}}\left(S_{m}\right)\right\|_{\sigma} \leq \varepsilon$ for $m \in\{1, \ldots, n\}$. Therefore, $T_{B_{i}^{*}, A_{i}^{*}}\left(\mathcal{C}_{\mathcal{D} \mathcal{I} \mathcal{R}}^{*}\right)$ is totally bounded and $T_{B_{i}^{*}}, A_{i}^{*}$ is a totally bounded operator.

Proposition 3.3. Let $T_{A_{i}, B_{i}}: \mathcal{A}_{\mathcal{D I R}} \rightarrow \mathcal{A}_{\mathcal{D I R}}$ be a linear map which is surjective up to compact operators. Then $T_{A_{i}, B_{i}}$ is essentially spectrally bounded if and only if $T_{A_{i}, B_{i}}\left(\mathcal{K}_{D I R}\right) \subseteq \mathcal{K}_{D I R}$ and the induced map $\widehat{T}_{A_{i}, B_{i}}: \mathcal{Q}_{D I R} \rightarrow \mathcal{Q}_{D I R}$ defined by $\widehat{T}_{A_{i}, B_{i}}(\varphi(X))=\varphi\left(T_{A_{i}, B_{i}}(X)\right) \forall X \in \mathcal{A}_{\mathcal{D I R}}$ is either a continuous automorphism or continuous anti-automorphism multiplied by nonzero scalar. 
Proof. Without loss of generality, assume that $T_{A_{i}, B_{i}}$ is essentially spectrally bounded such that $r_{e}\left(T_{A_{i}, B_{i}}(X)\right) \leq M r_{e}(X)$, for all $X \in \mathcal{A}_{\mathcal{D I R}}$ holds. It is sufficient to prove that the operator $T_{A_{i}, B_{i}}$ leaves the $\mathcal{K}_{\mathcal{D I R}}$ invariant. Let $E \in \mathcal{K}_{\mathcal{D I R}}$, we show that $T_{A_{i}, B_{i}}(E) \in \mathcal{K}_{\mathcal{D I R}}$. Let $S \in \mathcal{A}_{\mathcal{D I R}}$, since $T_{A_{i}, B_{i}}$ is surjective up to compact operators then there exists $Y^{\prime} \in \mathcal{A}_{\mathcal{D I R}}$ and $E^{\prime} \in \mathcal{A}_{\mathcal{D I R}}$ for which $S=T_{A_{i}, B_{i}}\left(S^{\prime}\right)+E^{\prime}$ and so $S^{\prime} \in \mathcal{A}_{\mathcal{D I R}}$. Now, $S+T_{A_{i}, B_{i}}(E)=T_{A_{i}, B_{i}}\left(S^{\prime}\right)+E^{\prime}+T_{A_{i}, B_{i}}(E)=$ $T_{A_{i}, B_{i}}\left(S^{\prime}+E\right)+E^{\prime} \in \mathcal{A}_{\mathcal{D I R}}$. Thus, for every $S \in A_{D I R}, S+T_{A_{i} . B_{i}}(E) \in \mathcal{A}_{\mathcal{D I R}}$. It follows that for every $E \in \mathcal{K}_{\mathcal{D I R}}$ we have $S+E \in \mathcal{A}_{\mathcal{D I R}}$ thus $T_{A_{i}, B_{i}}(E) \in \mathcal{A}_{\mathcal{D I R}}$ and we conclude that $T_{A_{i}, B_{i}}\left(\mathcal{K}_{D I R}\right) \subseteq \mathcal{K}_{D I R}$. Let $E \in \mathcal{K}_{\mathcal{D I R}}$ and $Y \in \mathcal{A}_{\mathcal{D I R}}$. We show that $T_{A_{i}, B_{i}}$ is surjective to compact operators, that is, $T_{A_{i}, B_{i}}\left(\mathcal{A}_{\mathcal{D I R}}\right)=\operatorname{Ran}\left(T_{A_{i}, B_{i}}\right)+\mathcal{K}_{\mathcal{D I R}}$. Therefore, for any $Y \in \mathcal{A}_{\mathcal{D I R}}$, there exists $C_{0} \in \mathcal{K}_{\mathcal{D I R}}$ such that $Y=T_{A_{i}, B_{i}}(X)+C_{0}$. Therefore,

$$
\begin{aligned}
r\left(\lambda \varphi\left(T_{A_{i}, B_{i}}(C)\right)+\varphi(Y)\right) & =r\left(\varphi\left(\lambda\left(T_{A_{i}, B_{i}}(C)\right)+Y\right)\right)=r_{e}\left(\lambda\left(T_{A_{i}, B_{i}}(C)\right)+Y\right)=r_{e}\left(T_{A_{i}, B_{i}}(\lambda C+X)+C_{0}\right) \\
& =r_{e}\left(T_{A_{i}, B_{i}}(\lambda C+X)\right) \leq M r_{e}(\lambda C+X)=M r_{e}(X), \lambda \in \mathbb{C} .
\end{aligned}
$$

This $\lambda \rightarrow r\left(\varphi\left(T_{A_{i}, B_{i}}(C)\right)+\varphi(Y)\right)$ is a subharmonic function on $\mathbb{C}$. By liouville's Theorem implies that $r\left(\varphi\left(T_{A_{i}, B_{i}}(C)\right)+\right.$ $\varphi(Y))=r(\varphi(Y))$. By Zemánek's characterization of the radical, we have $\varphi\left(T_{A_{i}, B_{i}}(C)\right)=0$ and $T_{A_{i}, B_{i}}(C) \in \mathcal{K}_{\mathcal{D I R}}$. This shows that $T_{A_{i}, B_{i}}\left(\mathcal{K}_{\mathcal{D I R}}\right) \subseteq \mathcal{K}_{\mathcal{D I R}}$. Thus, $T_{A_{i}, B_{i}}$ induces a surjective spectrally bounded linear map $\widehat{T}_{A_{i}, B_{i}}: \mathcal{Q}_{\mathcal{D I R}} \rightarrow \mathcal{Q}_{\mathcal{D I R}}$ defined by $\widehat{T}_{A_{i}, B_{i}}(\varphi(X))=\varphi\left(T_{A_{i}, B_{i}}(X)\right), \forall X \in \mathcal{A}_{\mathcal{D I R}}$. We show that $\widehat{T}_{A_{i}, B_{i}}$ is a continuous Jordan automorphism $\mathcal{U}$ multiplied by an invertible central element of $\mathcal{Q}_{\mathcal{D I R}}$. Now, consider a non-zero projection $P \in \mathcal{A}_{\mathcal{D I R}}$ where $\mathcal{A}_{\mathcal{D I R}}$ is a purely infinite $C^{*}$-algebra. It follows that $P \mathcal{A}_{\mathcal{D I R}} P$ is also properly infinite $C^{*}$-algebra and each element can be written as a finite sum of $P \mathcal{A}_{\mathcal{D I R}} P$. Thus

$$
T_{A_{i}, B_{i}}(P)+T_{A_{i}, B_{i}}(Q)+T_{A_{i}, B_{i}}(Q) T_{A_{i}, B_{i}}(P)=0, \forall Q \in \mathcal{A}_{\mathcal{D I} \mathcal{R}} .
$$

Suppose we replace $Q$ with $I-P$ then,

$$
T_{A_{i}, B_{i}}(P)+T_{A_{i}, B_{i}}(I)+T_{A_{i}, B_{i}}(I) T_{A_{i}, B_{i}}(P)=2\left(T_{A_{i}, B_{i}}(P)\right)^{2} .
$$

Multiplying the identity $T_{A_{i}, B_{i}}(I)$ on left hand side and $T_{A_{i}, B_{i}}(P)$ on the right hand side of Equation (3) and comparing the two equations

$$
T_{A_{i}, B_{i}}(I)\left(T_{A_{i}, B_{i}}(P)\right)^{2}=\left(T_{A_{i}, B_{i}}(P)\right)^{2} T_{A_{i}, B_{i}}(I) .
$$

Now, let $X=\sum_{i=1}^{n} \lambda_{i} P_{i}$ be a linear combination of orthogonal family of projection $P_{1}, \ldots, P_{n}$ of $\mathcal{A}_{\mathcal{D I R}}$. Applying Equation (2), we have $T_{A_{i}, B_{i}}(X)^{2}=\sum_{i=1}^{n} \lambda_{i}^{2} P_{i}^{2}$ and $\left(T_{A_{i}, B_{i}}(X)\right)^{2} T_{A_{i}, B_{i}}(I)=T_{A_{i}, B_{i}}(I)\left(T_{A_{i}, B_{i}}(X)\right)^{2}$ follows from Equation (4). Consequently $T_{A_{i}, B_{i}}$ is continuous and has real rank zero, thus $\left(T_{A_{i}, B_{i}}(M)\right)^{2} T_{A_{i}, B_{i}}(I)=T_{A_{i}, B_{i}}(I)\left(T_{A_{i}, B_{i}}(M)\right)^{2}$ holds for self-adjoint elements of $\mathcal{A}_{\mathcal{D I R}}$. Suppose we choose $Y \in \mathcal{B}_{\mathcal{D I R}}$ such that each element can be expressed as finite sum square zero elements in $\mathcal{B}_{\mathcal{D I R}}$ and $T_{A_{i}, B_{i}}$ is surjective and $T_{A_{i}, B_{i}}(I)$ is the central element of $\mathcal{B}_{\mathcal{D I R}}$. Using Equation (3) we have

$$
\left(T_{A_{i}, B_{i}}(P)\right)^{2}=T_{A_{i}, B_{i}}(P) T_{A_{i}, B_{i}}(I)=\left(T_{A_{i}, B_{i}}\left(P^{2}\right)\right) T_{A_{i}, B_{i}}(I), \forall \in \mathcal{A}_{\mathcal{D I R}} .
$$

Suppose that $N \in \mathcal{A}_{\mathcal{D I R}}$ such that $T_{A_{i}, B_{i}}(N)=I$ with $T_{A_{i}, B_{i}}(I)$ being invertible then

$$
I=\left(T_{A_{i}, B_{i}}(N)\right)^{2}=\left(T_{A_{i}, B_{i}}\left(N^{2}\right)\right)\left(T_{A_{i}, B_{i}}(I)\right)=\left(T_{A_{i}, B_{i}}(I)\right)\left(T_{A_{i}, B_{i}}\left(N^{2}\right)\right) .
$$

Let $\mathcal{U}(X)=\left(T_{A_{i}, B_{i}}(I)^{-1}\right)\left(T_{A_{i}, B_{i}}(X)\right), \forall X \in \mathcal{A}_{\mathcal{D I R}}$ and for all $X \in \mathcal{A}_{\mathcal{D I R}}$,

$$
(\mathcal{U}(X))^{2}=T_{A_{i}, B_{i}}(I)^{-2}\left(T_{A_{i}, B_{i}}(X)\right)^{2}=\left(T_{A_{i}, B_{i}}(I)^{-1}\right)\left(T_{A_{i}, B_{i}}\left(X^{2}\right)\right)=\mathcal{U}\left(X^{2}\right) .
$$

Hence $\mathcal{U}$ is a Jordan automorphism multiplied by an invertible central element of $\mathcal{Q}_{\mathcal{D I R}}$ and the centre $\widehat{T}_{A_{i}, B_{i}}(\varphi(X))=$ $\varphi\left(T_{A_{i}, B_{i}}(X)\right)$. Therefore, $\mathcal{Q}_{\mathcal{D I R}}$ is a prime $C^{*}$-subalgebras and $\mathcal{U}$ is a Jordan an automorphism or an anti-automorphism.

Theorem 3.5. Let $T_{A_{i}, B_{i}}: \mathcal{A}_{\mathcal{D I R}} \rightarrow \mathcal{B}_{\mathcal{D I R}}$ be a linear map which is surjective up to compact operators. Then $T_{A_{i}, B_{i}}$ compresses the essential spectrum if and only if $T_{A_{i}, B_{i}}\left(\mathcal{K}_{\mathcal{D I R}}\right) \subseteq \mathcal{K}_{\mathcal{D I R}}$ and the induced map $\widehat{T}_{A_{i}, B_{i}}: \mathcal{Q}_{\mathcal{D I R}} \rightarrow \mathcal{Q}_{\mathcal{D I R}}$ defined by $\widehat{T}_{A_{i}, B_{i}}(\varphi(X))=\varphi\left(T_{A_{i}, B_{i}}(X)\right) \forall X \in \mathcal{A}_{\mathcal{D I R}}$ is either a continuous automorphism or continuous anti-automorphism.

Proof. From Proposition 3.3, it follows that $T_{A_{i}, B_{i}}$ is an essentially spectrally bounded, that is, $r_{e}\left(T_{A_{i}, B_{i}}(X)\right) \leq M r_{e}(X)$ for all $X \in \mathcal{A}_{\mathcal{D I R}}$ and $T_{A_{i}, B_{i}}\left(\mathcal{K}_{\mathcal{D I R}}\right) \subseteq \mathcal{K}_{\mathcal{D I R}}$. Therefore, the induced map $\widehat{T}_{A_{i}, B_{i}}: \mathcal{Q}_{\mathcal{D I R}} \rightarrow \mathcal{Q}_{\mathcal{D I R}}$ is either a continuous automorphism or continuous anti-automorphism multiplied by a non-zero constant $\alpha$. Then $T_{A_{i}, B_{i}}$ is said to compress the essential spectrum if $\{\alpha\}=\sigma_{e}\left(T_{A_{i}, B_{i}}(X)\right) \subseteq \sigma_{e}(X)=I$ where $\alpha=I$. 
Proposition 3.4. Let $\mathcal{I}$ and $\mathcal{J}$ be two closed proper ideals of dense irreducible subalgebras $\mathcal{A}_{\mathcal{D I R}}$ and $\mathcal{B}_{\mathcal{D I R}}$ respectively such that $\mathcal{B}_{\mathcal{D I R}} / \mathcal{J}$ is semi-simple. Then for a linear mapping $T_{A_{i}, B_{i}}: \mathcal{A}_{\mathcal{D I R}} \rightarrow \mathcal{B}_{\mathcal{D I R}}$ the following conditions are equivalent:

(i). $T_{A_{i}, B_{i}}$ is essentially spectrally bounded and surjective modulo $\mathcal{J}$.

(ii). $T_{A_{i}, B_{i}} \mathcal{I} \subseteq \mathcal{J}$ and $\widehat{T}_{A_{i}, B_{i}}: \mathcal{A}_{\mathcal{D I R}} / \mathcal{I} \rightarrow \mathcal{B}_{\mathcal{D I R}} / \mathcal{J}$ spectrally bounded and surjective.

Proof. (ii) $\rightarrow$ (i). Since $T_{A_{i}, B_{i}}$ is spectrally compact then $T_{A_{i}, B_{i}} \mathcal{I} \subseteq \mathcal{J}$. We say that $T_{A_{i}, B_{i}}$ is essentially spectrally bounded if for any positive number $M \geq 0$, then $r\left(T_{A_{i}, B_{i}}(X)+\mathcal{J}\right) \leq M r(X+I)$, for all $X \in \mathcal{A}_{\mathcal{D I R}}$. Without the loss of generality, we assume that $\widehat{T}_{A_{i}, B_{i}}$ essentially spectrally bounded, that is, for all $X \in \mathcal{A}_{\mathcal{D I R}}, r\left(\widehat{T}_{A_{i}, B_{i}}(X)+\mathcal{J}\right) \leq M r(X+I)$. We see that $T_{A_{i}, B_{i}}$ is surjective modulo $\mathcal{J}$ if for every $Y \in \mathcal{B}_{\mathcal{D I R}}$ then, there is $X \in \mathcal{A}_{\mathcal{D I R}}$ such that $Y-T_{A_{i}, B_{i}}(X) \in \mathcal{J}$. Thus, for every $Y \in \mathcal{B}_{\mathcal{D I R}}$ then $Y+\mathcal{J}=\widehat{T}_{A_{i}, B_{i}}(X+I)=T_{A_{i}, B_{i}}(X)+J$ for some $X \in \mathcal{A}_{\mathcal{D I R}}$ hence $T_{A_{i}, B_{i}}$ is surjective modulo $\mathcal{J}$.

(i) $\Rightarrow$ (ii). Now, we prove that $T_{A_{i}, B_{i}} \mathcal{I} \subseteq \mathcal{J}$, then fix $X \in \mathcal{I}$ and $Y \in \mathcal{B}_{\mathcal{D I R}}$ such that there exists $X_{1} \in \mathcal{A}_{\mathcal{D I R}}$ such that $Y-T_{A_{i}, B_{i}}\left(X_{1}\right) \in \mathcal{J}$. For any $\lambda \in \mathbb{C}$, it holds that

$$
r\left(\lambda\left(T_{A_{i}, B_{i}}(X)+\mathcal{J}\right)\right)+Y+\mathcal{J}=r\left(\lambda T_{A_{i}, B_{i}}(X)+Y+\mathcal{J}\right)=r\left(\lambda T_{A_{i}, B_{i}}\left(X+X_{1}\right)+\mathcal{J}\right) \leq M r\left(\lambda X+X_{1}+\mathcal{I}\right)=\operatorname{Mr}\left(X_{1}+\mathcal{I}\right) .
$$

for some constant $M \geq 0$. Using the sub-harmonicity of spectral radius function $\lambda \rightarrow r\left(\lambda\left(T_{A_{i}, B_{i}}(X)+\mathcal{J}\right)\right)+\mathcal{Y}+\mathcal{J}$ is bounded on $\mathbb{C}$ hence constant and $r\left(T_{A_{i}, B_{i}}(X)+\mathcal{J}+Y+\mathcal{J}\right)=r(Y+\mathcal{J})$, for all $Y \in \mathcal{B}_{\mathcal{D I R}}$. Thus, $T_{A_{i}, B_{i}}(X)+\mathcal{J} \in \operatorname{Rad}\left(\mathcal{B}_{\mathcal{D I R}}\right) / \mathcal{J}$ and by Zamanek's characterization of the radical $T_{A_{i}, B_{i}}(X) \in \mathcal{J}$ is semi-simple.

Proposition 3.5. Let $\mathcal{I}$ and $\mathcal{J}$ be two closed proper ideals of dense irreducible subalgebras $\mathcal{A}_{\mathcal{D I R}}$ and $\mathcal{B}_{\mathcal{D I R}}$ respectively such that $\mathcal{B}_{\mathcal{D I R}} / \mathcal{J}$ is semi-simple. If $T_{A_{i}, B_{i}}: \mathcal{A}_{\mathcal{D I R}} \rightarrow \mathcal{B}_{\mathcal{D I R}}$ is essentially spectrally bounded and surjective modulo $\mathcal{J}$, then $T_{A_{i}, B_{i}}$ is a Jordan automorphism modulo $\mathcal{J}$.

Proof. Suppose that $\mathcal{A}_{\mathcal{D I R}}$ be a $C^{*}$-subalgebra with real rank zero and $\mathcal{B}_{\mathcal{D I R}}$ be a unital Banach algebra. Then by Proposition 3.4, $T_{A_{i}, B_{i}} \mathcal{I} \subseteq \mathcal{J}$ and $\widehat{T}_{A_{i}, B_{i}}: \mathcal{A}_{\mathcal{D I R}} / \mathcal{I} \rightarrow \mathcal{B}_{\mathcal{D I R}} / \mathcal{J}$ is unital essentially spectrally bounded compact and surjective. Suppose that $P$ is a nonzero projection in $\mathcal{A}_{\mathcal{D I} \mathcal{R}}$. It follows that $P$ is a properly infinite since $\mathcal{A}_{\mathcal{D I R}}$ is a purely infinite and the hereditary property of $C^{*}$ - subalgebras implies that $P \mathcal{A}_{\mathcal{D I R}} P$ is properly infinite. In fact, the projection $P \mathcal{A}_{\mathcal{D I R}} P$ can be expressed as a finite sum of square zero elements in $P \mathcal{A}_{\mathcal{D I R}} P$. Now, $T_{A_{i}, B_{i}}$ sends every projection in $\mathcal{A}_{\mathcal{D I R}}$ into an idempotent in $\mathcal{B}_{\mathcal{D I R}}$. Thus, choose any $X \in \mathcal{A}_{\mathcal{D I R}}$ with $X^{2}=0$ then $\left(T_{A_{i}, B_{i}}(X)\right)^{2}=0$ hence $T_{A_{i}, B_{i}}$ is Jordan automorphism because the linear span of orthogonal projection is dense in $\mathcal{A}_{\mathcal{D I R}}$ and thus rank zero. It follows that $\widehat{T}_{A_{i}, B_{i}}$ is Jordan automorphism modulo $\mathcal{J}$.

Lemma 3.2. Let $T_{A_{i}, B_{i}}: \mathcal{A}_{\mathcal{D I R}} \rightarrow \mathcal{B}_{\mathcal{D I R}}$ be a spectrally bounded compact elementary operator from a unital dense irreducible $C^{*}$-subalgebra $\mathcal{A}_{\mathcal{D I R}}$ into unital semi-simple dense irreducible Banach subalgebra $\mathcal{B}_{\mathcal{D I R}}$. If $P, Q$ of mutually orthogonal projections in $\mathcal{A}_{\mathcal{D I R}}$ then

$$
\left(T_{A_{i}, B_{i}}(X)\right)\left(T_{A_{i}, B_{i}}(Y)\right)+\left(T_{A_{i}, B_{i}}(Y)\right)\left(T_{A_{i}, B_{i}}(X)\right)=0, \text { for every } X \in P \mathcal{A}_{\mathcal{D I R}} P, Y \in Q \mathcal{A}_{\mathcal{D I R}} Q
$$

Proof. Because of Proposition 3.5, $T_{A_{i}, B_{i}}$ preserves square zero and is spectrally bounded. Suppose that $X \in P \mathcal{A}_{\mathcal{D I R}} P$ and $Y \in Q \mathcal{A}_{\mathcal{D I R}} Q$ then each element can be written as a finite sum $X=\sum_{i} X_{i}$ and $Y=\sum_{j} Y_{j}$, respectively, where $\sum_{i} X_{i} \in P \mathcal{A}_{\mathcal{D I R}} P, \sum_{j} Y_{j} \in Q \mathcal{A}_{\mathcal{D I R}} Q$ are square zero for all $i, j$. This means that $\left(X_{i}+Y_{j}\right)^{2}=0$ and $\left(T_{A_{i}, B_{i}}\left(X_{i}+Y_{j}\right)\right)^{2}=0$ which yields,

$$
\left(T_{A_{i}, B_{i}}\left(X_{i}\right)\right)\left(T_{A_{i}, B_{i}}\left(Y_{i}\right)\right)+\left(T_{A_{i}, B_{i}}\left(Y_{i}\right)\right)\left(T_{A_{i}, B_{i}}\left(X_{i}\right)\right)=0 .
$$

Summing over all $i, j$ we have $\left(T_{A_{i}, B_{i}}(X)\right)\left(T_{A_{i}, B_{i}}(Y)\right)+\left(T_{A_{i}, B_{i}}(Y)\right)\left(T_{A_{i}, B_{i}}(X)\right)=0$.

Theorem 3.6. Let $T_{A_{i}, B_{i}}: \mathcal{A}_{\mathcal{D I R}} \rightarrow \mathcal{B}_{\mathcal{D I R}}$ be a spectrally bounded compact elementary operator from a unital dense irreducible $C^{*}$-subalgebra $\mathcal{A}_{\mathcal{D I R}}$ onto semi-simple dense irreducible Banach subalgebra $\mathcal{B}_{\mathcal{D I R}}$. If $T_{A_{i}, B_{i}}(I)$ is an invertible element in centre of $\mathcal{B}_{\mathcal{D I R}}$ then every nonzero projection in $\mathcal{A}_{\mathcal{D I R}}$ is properly infinite.

Proof. Let $P \in \mathcal{A}_{\mathcal{D I R}}$ be a nontrivial projection. Suppose that $X=P$ and $Y=Q=I-P$. Using Equation (5), we have

$$
\left(T_{A_{i}, B_{i}}(P)\right) T_{A_{i}, B_{i}}(I-P)+T_{A_{i}, B_{i}}(I-P)\left(T_{A_{i}, B_{i}}(P)\right)=0 .
$$

Since $\left(T_{A_{i}, B_{i}}(P)\right)^{2}=\left(T_{A_{i}, B_{i}}(P)\right)$ then substituting $T_{A_{i}, B_{i}}(I-P)$ with $T_{A_{i}, B_{i}}(I)$, we have

$$
\left(T_{A_{i}, B_{i}}(P)\right)\left(T_{A_{i}, B_{i}}(I)\right)+\left(T_{A_{i}, B_{i}}(I)\right)\left(T_{A_{i}, B_{i}}(P)\right)=2\left(T_{A_{i}, B_{i}}(P)\right)^{2}
$$


By multiplying the identity $\left.T_{A_{i}, B_{i}}(I)\right)$ on the left and on the right by $T_{A_{i}, B_{i}}(P)$ and subtracting the resulting identities, we obtain

$$
\left(T_{A_{i}, B_{i}}(P)^{2}\left(T_{A_{i}, B_{i}}(I)\right)=\left(T_{A_{i}, B_{i}}(I)\right) T\left({ }_{A_{i}, B_{i}} P\right)^{2},\right.
$$

for nontrivial projection $P$. Suppose $\left\{P_{1}, \ldots, P_{n}\right\}$ is an orthogonal family in $\mathcal{A}_{\mathcal{D I R}}$ and $\lambda_{1}, \ldots, \lambda_{n} \in \mathbb{R}$ and by applying Equation (5), $\left(T_{A_{i}, B_{i}}\left(\sum_{i} \lambda_{i} P_{i}\right)\right)^{2}=\sum_{i} \lambda_{i}^{2}\left(T_{A_{i}, B_{i}}(P)_{i}\right)^{2}$. Also, $\mathcal{A}_{\mathcal{D I R}}$ has a real rank zero, therefore the linear combinations are dense in a subset of self-adjoint elements of $\mathcal{A}_{\mathcal{D I R}}$. In fact, $T_{A_{i}, B_{i}}$ is spectrally bounded and it extends to all $X$ which are selfadjoint in $\mathcal{A}_{\mathcal{D I R} \mathcal{R}}$. Now $X Y+Y X=(X+Y)^{2}-X^{2}-Y^{2}$ and $(X+i Y)^{2}=X^{2}-Y^{2}+i(X Y+Y X)$ for all self-adjoint $X, Y \in \mathcal{A}_{\mathcal{D I R}}$. We conclude that $T_{A_{i}, B_{i}}(I)$ commutes with $\left(T_{A_{i}, B_{i}}(W)\right)^{2}$ for every $W \in \mathcal{A}_{\mathcal{D I R}}$. Then $T_{A_{i}, B_{i}}$ is surjective and $T_{A_{i}, B_{i}}(I)$ commutes with every square of an element $\mathcal{B}_{\mathcal{D I R}}$ but since $2 Y=(I+Y)^{2}-I-Y^{2}$ for each $Y \in \mathcal{B}_{\mathcal{D I R}}$. We obtain $T_{A_{i}, B_{i}}(I)$ belong to centre of $\mathcal{B}_{\mathcal{D I R}}$. Consequently $T_{A_{i}, B_{i}}\left(P^{2}\right)\left(T_{A_{i}, B_{i}}(I)\right)=T_{A_{i}, B_{i}}(P)\left(T_{A_{i}, B_{i}}(I)\right)=\left(T_{A_{i}, B_{i}}(P)^{2}\right.$ for every projection $P \in \mathcal{A}_{\mathcal{D I R}}$. Thus, $\mathcal{A}_{\mathcal{D I R}}$ has a rank zero, we see that $\left(T_{A_{i}, B_{i}}(X)\right)^{2}\left(T_{A_{i}, B_{i}}(I)\right)=\left(T_{A_{i}, B_{i}}(X)\right)^{2}$, for every $X \in \mathcal{A}_{\mathcal{D I R}}$. Since $T_{A_{i}, B_{i}}$ is surjective, it follows that $T_{A_{i}, B_{i}}(I)$ must be invertible.

\section{Open problems}

Most of the linear mappings on Banach algebras are elementary operators. Due to varied definitions of elementary operators, the interplay between the structural properties of elementary operators and the underlying algebra is often challenging; for example, the structure of spectrally bounded compact elementary operators on general Banach algebras. We end this paper by stating the following open problems.

(i). Establish some properties of power bounded spectrally compact elementary operators on general Banach subalgebras.

(ii). Investigate the idempotency and orthogonality properties of spectrally bounded compact elementary operators on general Banach subalgebras.

\section{References}

[1] C. A. Akemann, S. Wright, Compact and weakly compact derivations of $C^{*}$-algebras, Pacific J. Math. 85 (1979) 253-259.

[2] P. Ara, M. Mathieu, Local Multiplier of $C^{*}$-algebras, Springer, London, 2003.

[3] M. Bresar, Y. V. Turovskii, Compactness conditions for elementary operators, Stud. Math. 178 (2007) 1-18.

[4] J. B. Conway, A Course in Operator Theory, American Mathematical Society, Providence, 2000.

[5] R. Curto, M. Mathieu, Spectrally bounded generalized inner derivations, Proc. Amer. Math. Soc. 123 (1995) 2431-2434.

[6] R. Curto, M. Mathieu, Elementary Operators and Their Applications, Birkhauser, Belfast, 2009.

[7] G. Dales, P. Aiena, J. Eschmier, K. B. Laursen, G. Willis, Introduction to Banach Algebras, Operators and Harmonic Analysis, Cambridge Univ. Press, New York, 2003.

[8] H. K. Du, Y. Q. Wang, G. B. Gao, Norms of elementary operators, Proc. Amer. Math. Soc. 36 (2008) 1337-1348.

[9] L. A. Fialkow, Spectral properties of elementary operators II, Trans. Amer. Math. Soc. 290 (1985) 415-429.

[10] K. Fong, A. R. Sourour, On the operator identity $\sum A_{k} X B_{k}=0$, Canad. J. Math. 31 (1979) 845-857.

[11] Y. F. Jung, Spectrally bounded jordan derivations on banach algebras, Bull. Korean Math. Soc. 36 (1993) 477-481.

[12] E. Kreyzig, Introductory Functional Analysis with Applications, Wiley, New York, 1978.

[13] Y. F Lin, M. Mathieu, Jordan isomorphisms of purely infinite $C^{*}$-algebras, Quart. J. Math. 58 (2007) 249-253.

[14] G. Lumer, M. Rosenblum, Linear operator equations, Proc. Amer. Math. Soc. 10 (1959) 32-41.

[15] M. Mathieu, Where is the image of derivation, Banach Center Publ. 30 (1994) 237-249.

[16] M. Mathieu, Spectrally bounded traces on $C^{*}$-algebras, Bull. Aust. Math. Soc. 68 (2003) 169-173.

[17] M. Mathieu, A collection of problems on spectrally bounded operators, Asian-Eur. J. Math. 2 (2009) 487-501.

[18] M. Mathieu, G. J. Schick, First results on spectrally bounded operators, Stud. Math. 152 (2002) 187-198.

[19] M. Mathieu, Elementary operators still not elementary, Opuscula Math. 36 (2016) 787-797.

[20] T. Huruya, J. Tomiyama, Completely bounded maps of $C^{*}$-algebras, J. Operator Theory 10 (1983) 141-152.

[21] N. B. Okelo, J. O. Agure, D. O. Ambogo, Norms of elementary operators and characterization of norm-attainable operators, Int. J. Math. Anal. 24 (2010) 1197-1204.

[22] N. B. Okelo, J. O. Agure, A two-sided multiplication operator norm, Gen. Math. Notes 2 (2011) 18-23.

[23] N. B. Okelo, J. O. Agure, P. O. Oleche, Various notions of orthogonality in normed spaces, Acta Math. Sci. 33 (2013) $1387-1397$.

[24] N. B. Okelo, On orthogonality of elementary operators in norm-attainable classes, Taiwanese J. Math. 24 (2020) 119-130,

[25] T. W. Palmer, Banach Algebras and the General Theory of *-Algebras, Cambridge Univ. Press, New York, 1994.

[26] V. Ptak, Derivations, commutators and radical, Manuscripta Math. 23 (1978) 355-362.

[27] E. Saksman, H. Tylli, Multiplications and elementary operators in the Banach space setting, In: J. M. F. Castillo, W. B. Johnson, Methods in Banach Space Theory, London Math. Soc. Lecture Note Ser. 337, Cambridge Univ. Press, Cambridge, 2006, pp. 253-292.

[28] P. Semrl, Spectrally bounded maps on B(H), Quart. J. Math. 49 (1998) 87-92.

[29] V. S. Shulman, L. Turowska, An Elementary Approach to Elementary Operators on $B(H)$, Springer, Basel, 2011.

[30] J. G. Stampfli, The norm of a derivation, Pacific J. Math. 33 (1970) 737-747.

[31] G. P. Tripathi, Compactness and weak compactness of elementary operators on $B l^{2}$ induced by composition operators on $l^{2}, M a t . B e c h n k$ 61 (2009) 227-233.

[32] K. Vala, On compact sets of compact operators, Ann. Acad. Sci. Fenn. Math. 351 (1964) 1-9. 